Dipartimento di Matematica, Università di Trento

Preprint UTM - 737

\title{
A variational approach \\ to stochastic nonlinear diffusion problems with dynamical boundary conditions
}

\author{
Stefano BONACCORSI, Giacomo ZIGLIO* \\ Dipartimento di Matematica, Università di Trento, \\ via Sommarive 14, 38123 Povo (Trento), Italia \\ stefano.bonaccorsi@unitn.it, ziglio@science.unitn.it
}

\begin{abstract}
We study a nonlinear partial differential equation of the calculus of variation in a bounded domain, perturbed by noise; we allow stochastic boundary conditions which depend on the time derivative of the solution on the boundary. This work provides the existence and uniqueness of the solution and it shows the existence of an ergodic invariant measure for the corresponding transition semigroup; further, under suitable additional assumptions, uniqueness and strong asymptotic stability of the invariant measure are proved.

Keywords: Nonlinear operators, dynamical boundary conditions, invariant measures, ergodicity
\end{abstract}

1991 MSC: $60 \mathrm{H} 15,58 \mathrm{~F} 11,47 \mathrm{H} 15$

\section{Introduction}

Let $\mathcal{O} \subset \mathbb{R}^{n}$ be a bounded smooth domain with regular boundary $\Gamma=\partial \mathcal{O}$; throughout the whole paper we fix $2<p<\infty$ and $T>0$. We consider in $\mathcal{O}$ the stochastic nonlinear diffusion equation

$$
\mathrm{d} u(t, x)=\operatorname{div} \mathbf{a}(x, \nabla u) \mathrm{d} t+b \cdot \mathrm{d} w(t, x) \quad \text { in } \mathcal{O}
$$

where $\mathbf{a}$ is a differential operator of Leray-Lions type, i.e., $\mathbf{a}: \mathcal{O} \times \mathbb{R}^{n} \rightarrow \mathbb{R}^{n}$ is a Carathéodory function satisfying the conditions:

(a1) there exists $\delta>0$ such that $\mathbf{a}(x, \xi) \cdot \xi \geq \delta|\xi|^{p}$ for a.e. $x \in \mathcal{O}$ and for all $\xi \in \mathbb{R}^{n}$,

(a2) there exist $\sigma>0$ and $\rho \in L^{q}(\mathcal{O})$ such that $|\mathbf{a}(x, \xi)| \leq \sigma\left(\rho(x)+|\xi|^{p-1}\right)$ for a.e. $x \in \mathcal{O}$ and for all $\xi \in \mathbb{R}^{n}$, where $q=p /(p-1)$,

(a3) for a.e. $x \in \mathcal{O}$ and for all $\xi \neq \eta \in \mathbb{R}^{n}$ :

$$
(\mathbf{a}(x, \xi)-\mathbf{a}(x, \eta)) \cdot(\xi-\eta)>0 .
$$

${ }^{*}$ The research of G. Ziglio was supported by Project NeSt funded by Provincia Autonoma di Trento (P.A.T.) within Bando unità di ricerca 2006 
Such conditions are classical in the literature, compare [13, §2] or [14, Ch. 2] for a more general survey. Note that operators such as the $p$-Laplacian are included in our model by choosing $\mathbf{a}(x, \xi)=|\xi|^{p-2} \xi$.

When $w=0$, equation (1.1) is used as a model of diffusion in several fields, such as heat propagation in a plasma gas, population dynamics and other examples of nonlinear diffusive phenomena. Typical derivation of (1.1) comes from nonlinear version of Darcy's law

$$
J(t, x)=-\frac{1}{\mu} k(\rho \nabla p) \rho \nabla p
$$

where $J(t, x)$ is the vector flux rate, $\rho(t, x)$ is the density and $p(t, x)$ the pressure of the fluid. Physically, the above version of Darcy's law occours when the permeability is assumed to be depending on the flux; compare the discussion in [18, §A.2].

As opposite to the larger part of the existing literature, we shall not associate to the nonlinear parabolic stochastic partial differential equation (1.1) standard, time-homogeneous boundary conditions of Dirichlet or Neumann type.

However, physical systems may be subject to random influences also through the boundary and this leads to stochastic dynamical boundary conditions. Dynamical means that they involves a time derivative of the solution on the boundary; namely, they have the form

$$
\mathrm{d} \tilde{u}(t, \xi)=\left[-\tilde{u}|\tilde{u}|^{p-2}(t, \xi)-\mathbf{a}(x, \nabla u) \cdot \nu\right] \mathrm{d} t+\tilde{b} \cdot \mathrm{d} \tilde{w}(t, \xi) \quad \text { on } \Gamma .
$$

Such kind of boundary conditions are already present in the literature, even for stochastic models, compare for instance [4]; they have a natural derivation in the description of phisical models with a dynamics on the boundary, such as heat transfer in a solid imbedded in a moving fluid $[20, \S 7.4]$, surface gravity waves in oceanic models $[8,10,16]$, problems in fluid dynamics [19], phase separation phenomena [9], etc.

In our model the noise (which represents the influence of a random heating source in the system) acts both in the domain and on the boundary and is presented as the temporal generalized derivative of an infinite-dimensional Wiener process taking values in the space $L^{2}(\mathcal{O}) \times L^{2}(\Gamma) . \quad b$ and $\tilde{b}$ are linear, trace class operators from $L^{2}(\mathcal{O})$ and $L^{2}(\Gamma)$ in themselves, respectively.

There are several different approaches in the literature to solve stochastic partial differential equations; in this paper, we shall follow the variational approach which allows a natural analysis of monotone, nonlinear problems. In Section 2 we briefly describe what is required for this approach to work, whereas we refer to $[12,17]$ for a complete survey on the variational approach to SPDEs.

The main result in Section 3, Theorem 5, guarantees the existence and uniqueness of a variational solution (in the sense of Definition 2) for the equation (1.1) with boundary conditions (1.2).

In Section 4 we investigate the asymptotic behaviour of the transition semigroup $P_{t}$ associated with the system (1.1)-(1.2). Using the approach explained in [3], based on the well known Krylov-Bogoliubov theorem, we prove existence of invariant measures (that is, strictly stationary solutions) and also of an ergodic one by means of the Krein-Milman theorem. 
Finally, under a super-linearity assumption on the Carathéodory function we show that the invariant measure is actually unique and strongly mixing. Such results on the strong asymptotic stability provide a fairly complete description of the asymptotic behaviour of solutions to the problem under study.

We also mention that the study of asymptotic properties for infinite-dimensional nonlinear SDEs driven by multiplicative Poisson noise has been considered in [15].

As it is known from the general theory, we can modify equations (1.1) and (1.2) by adding Lipschitz nonlinear operators (which may be $(t, \omega)$-dependent) acting both in the deterministic and the stochastic terms (which implies the case of nonconstant diffusion coefficients). In this case, it is possible to reformulate our results about existence of the solution and ergodic properties.

\section{The variational approach to stochastic equations}

In this section we shall introduce essential conditions in order to solve stochastic evolution equations in infinite dimensional Hilbert spaces of the form

$$
\mathrm{d} X(t)=A(t, X(t)) \mathrm{d} t+B(t, X(t)) \mathrm{d} W(t)
$$

where, in general, $A$ and $B$ are unbounded nonlinear operators in suitable Banach spaces, which may depend also on the elementary outcome $\omega$ in a non-anticipating fashion.

According to the problem (1.1) under consideration, we consider only the case of operators $A$ and $B$ independent of $t \in[0, T]$ and $\omega \in \Omega$. Therefore we are dealing with a SPDE of the following type

$$
\left\{\begin{aligned}
\mathrm{d} X(t) & =A(X(t)) \mathrm{d} t+B \mathrm{~d} W(t) \\
X(0) & =x_{0} \in H
\end{aligned}\right.
$$

where $H$ is a separable Hilbert space endowed with inner product $\langle\cdot, \cdot\rangle_{H}$ and norm $|\cdot|_{H}$, while $W(t)$ is a cylindrical Wiener process taking values in $H$.

The basic idea which makes this approach work is to construct/find a functional setting in order to the coefficients $A$ and $B$ of (2.1) satisfy certain monotonicity assumptions (see Hypothesis 1 below). In particular we need to find a reflexive Banach space $V$ (endowed with norm $\|\cdot\|_{V}$ ) continuously and densely embedded into $H$ so that we get a so called Gelfand triple, i.e. a triple of spaces $\left(V, H, V^{*}\right)$ such that

$$
V \hookrightarrow H\left(\equiv H^{*}\right) \hookrightarrow V^{*}
$$

where $H$ is identified with its dual $H^{*}$ via the Riesz isomorphism. Furthermore, in order to investigate the ergodic properties of the infinite-dimensional stochastic differential equation, we shall assume that $V \hookrightarrow H$ is compact. Let us stress the fact that the space $V$ has to be chosen carefully so that $A: V \rightarrow V^{*}$ satisfies the relevant monotonicity conditions stated below.

We let $L_{2}(H)$ denote the space of Hilbert-Schmidt operators from $H$ to itself, endowed with the norm $\|G\|_{H S}^{2}=\operatorname{Tr}\left(G G^{\star}\right)$. Then we require that $B \in L_{2}(H)$. 
Hypothesis 1 The operator A satisfies the following conditions.

(H1) $A$ is hemicontinuous: for all $u, v, x \in V$ the map

$$
\mathbb{R} \ni \lambda \rightarrow V^{*}\langle A(u+\lambda v), x\rangle_{V}
$$

is continuous.

(H2) $A$ is weakly monotonic: there exists $c \in \mathbb{R}$ such that for all $u, v \in V$ it holds

$$
V^{*}\langle A(u)-A(v), u-v\rangle_{V} \leq c|u-v|_{H}^{2} ;
$$

(H3) $A$ is coercive: there exists $\left.\alpha>1, c_{1} \in\right] 0, \infty\left[, c_{2} \in \mathbb{R}\right.$ such that for all $v \in V$ :

$$
V^{*}\langle A(v), v\rangle_{V} \leq-c_{1}\|v\|_{V}^{\alpha}+c_{2}|v|_{H}^{2}
$$

(H4) $A$ is bounded from $V$ into $V^{*}$ : there exists $c_{3} \in\left[0, \infty\left[\right.\right.$ and $c_{4} \in \mathbb{R}$ such that for all $v \in V$ :

$$
\|A(v)\|_{V^{*}} \leq c_{4}+c_{3}\|v\|_{V}^{\alpha-1}
$$

where $\alpha$ is as in (H3).

Remark 1 (H1)-(H2) imply that $A$ is demicontinuous, i.e.,

$$
\left\{v_{n}\right\} \subset V, v_{n} \rightarrow v \text { strongly in } V \quad \Longrightarrow \quad A\left(v_{n}\right) \rightarrow A(v) \text { weakly in } V^{*} .
$$

For a detailed proof see [17, Remark 4.1.1].

Under the hypotheses above, it is possible to formulate an existence and uniqueness result for solutions of variational type.

Definition 2 A continuous $H$-valued, $\left(\mathcal{F}_{t}\right)$-adapted process $\{X(t), t \in[0, T]\}$ is a variational solution of (2.1) if there exists a $\mathrm{d} t \otimes \mathbb{P}$-version $\hat{X}$ of $X$ such that $\hat{X}$ belongs to $L^{\alpha}([0, T] \times \Omega ; V) \cap L^{2}([0, T] \times \Omega ; H)$ with $\alpha$ as in $(H 3)$ and $\mathbb{P}$-a.s.

$$
X(t)=x_{0}+\int_{0}^{t} A(\bar{X}(s)) \mathrm{d} s+\int_{0}^{t} B \mathrm{~d} W(s), \quad t \in[0, T]
$$

where $\bar{X}$ is a $V$-valued progressively measurable $\mathrm{d} t \otimes \mathbb{P}$-version of $\hat{X}$.

Theorem 3 Let $A$ satisfy $(H 1)-(H 4)$ and $B \in L_{2}(H)$; let $x_{0} \in L^{2}\left(\Omega, \mathcal{F}_{0}, \mathbb{P} ; H\right)$ and $T \in[0, \infty)$ be fixed. Then there exists a unique variational solution $\{X(t), t \in$ $[0, T]\}$ of (2.1) and it holds, further, that

$$
\mathbb{E} \sup _{t \in[0, T]}|X(t)|_{H}^{2}<\infty .
$$

Moreover, the solution is a Markov process. 


\section{The existence and uniqueness result}

In this section we show that the model of stochastic nonlinear diffusion with dynamical boundary conditions introduced in Section 1 enters in the general framework outlined in Section 2.

We consider the problem

$$
\left\{\begin{array}{rlrl}
\mathrm{d} u(t, x) & =\operatorname{div} \mathbf{a}(x, \nabla u) \mathrm{d} t+b \mathrm{~d} w(t, x) & & \text { in } \mathcal{O} \\
u(t, \xi) & =\tilde{u}(t, \xi) \quad \text { when restricted to } \Gamma & \\
\mathrm{d} \tilde{u}(t, \xi) & =\left[-\tilde{u}(t, \xi)|\tilde{u}(t, \xi)|^{p-2}-\mathbf{a}(\xi, \nabla u) \cdot \nu\right] \mathrm{d} t+\tilde{b} \mathrm{~d} \tilde{w}(t, \xi) & & \text { on } \Gamma \\
u(0, x) & =u_{0}(x), \quad \tilde{u}(0, \xi)=\tilde{u}_{0}(\xi) . &
\end{array}\right.
$$

\subsection{Functional setting}

Given a bounded domain $\mathcal{O} \subset \mathbb{R}^{n}$ sufficiently regular (see e.g. [1, Remark 7.45]) we introduce the standard Sobolev spaces $W^{1, p}(\mathcal{O})$ for $1<p<\infty$, which will result the right choice as the functional space of functions defined on $\mathcal{O}$.

Further, we define the fractional order Sobolev spaces of functions on the boundary $\Gamma=\partial \mathcal{O}$

$$
W^{1-1 / p, p}(\Gamma)=\left\{u \in L^{p}(\Gamma) \mid \int_{\Gamma} \int_{\Gamma} \frac{|u(x)-u(y)|^{p}}{|x-y|^{p+N-2}} \mathrm{~d} \sigma(x) \mathrm{d} \sigma(y)<+\infty\right\}
$$

where $\mathrm{d} \sigma$ denotes the surface measure on $\partial \mathcal{O}$. The following result of compactness of the injection will be useful later:

$$
W^{1-1 / p, p}(\Gamma) \hookrightarrow L^{p}(\Gamma) \quad \text { compactly for all } p>1 \text {. }
$$

For a smooth domain $\mathcal{O}$ we define an operator trace, $L: W^{1, p}(\mathcal{O}) \rightarrow L^{p}(\Gamma)$ such that for any $u \in C(\overline{\mathcal{O}}) \cap W^{1, p}(\mathcal{O}), L u(\xi)=u(\xi)$ on $\Gamma$ is well defined.

It holds that the trace of functions in $W^{1, p}(\mathcal{O})$ are more regular than just $L^{p}(\Gamma)$. Actually it holds that

$$
L\left(W^{1, p}(\mathcal{O})\right)=W^{1-1 / p, p}(\Gamma),
$$

see [7, Proposition 3.31] or [1, Theorem 7.39], and denoting $\tilde{u}:=L u$ it holds

$$
\|\tilde{u}\|_{W^{1-1 / p, p}(\Gamma)} \leq K\|u\|_{W^{1, p}(\mathcal{O})}
$$

for some constant $K$ independent of $u$.

For a characterization of $W^{1-1 / p, p}(\Gamma)$ as the Besov space $B^{1-1 / p ; p, p}(\Gamma)$, i.e. as the intermediate space between $L^{p}(\Gamma)$ and $W^{1, p}(\Gamma)$, we refer to $[1, \S 7.6, \S 7.30, \S 7.38]$.

Let us start introducing as the "pivot" space the separable product Hilbert space

$$
H=\left\{\bar{u}:=(u, \tilde{u}) \in L^{2}(\mathcal{O}) \times L^{2}(\Gamma)\right\}
$$

endowed with the natural inner product

$$
\langle\bar{u}, \bar{v}\rangle_{H}=\langle u, v\rangle_{L^{2}(\mathcal{O})}+\langle\tilde{u}, \tilde{v}\rangle_{L^{2}(\Gamma)}
$$


for $\bar{u}=(u, \tilde{u}), \bar{v}=(v, \tilde{v}) \in H$ and norm $|\cdot|_{H}$. We shall identify $H$ with its dual $H^{*}$ via the Riesz isomorphism. Then, for $p>2$ we introduce the product Banach space

$$
V=\left\{\bar{v}:=(v, \tilde{v}) \in W^{1, p}(\mathcal{O}) \times W^{1-1 / p, p}(\Gamma) \mid v=\tilde{v} \text { on } \Gamma\right\}
$$

endowed with the norm (equivalent to that one in $W^{1, p}(\mathcal{O})$ thanks to the previous estimate on the boundary norm)

$$
\|\bar{v}\|_{V}=\|v\|_{L^{p}(\mathcal{O})}+\|\nabla v\|_{L^{p}(\mathcal{O})} .
$$

This norm is also equivalent to the following (for details see [11, footnote 4]):

$$
\|\bar{v}\|_{V}=\|\tilde{v}\|_{L^{p}(\Gamma)}+\|\nabla v\|_{L^{p}(\mathcal{O})} .
$$

The imbedding $V \hookrightarrow H$ is compact, thanks to the standard Sobolev's imbedding theorem [1, Theorem 6.3] and (3.2)-(3.3). Further, $V$ is reflexive since it is isomorphic to the reflexive Banach space $W^{1, p}(\mathcal{O})$ by the map $V \ni(u, \tilde{u}) \rightarrow u \in W^{1, p}(\mathcal{O})$.

Since $C^{\infty}(\overline{\mathcal{O}})$ is dense in $W^{1, p}(\mathcal{O})$ (compare [1, Theorem 3.16]) and using Sobolev's imbedding theorem, we obtain that the space

$$
W=\left\{\bar{u}=(u, \tilde{u}) \in C^{\infty}(\overline{\mathcal{O}}) \times C^{\infty}(\Gamma) \mid u=\tilde{u} \text { on } \Gamma\right\}
$$

is dense in $V$ and in $H$. We let $V^{*}$ be the dual space of $V$ under the dualization

$$
V^{*}\langle\bar{v}, \bar{u}\rangle_{V}=\langle\bar{v}, \bar{u}\rangle_{H} \quad \text { for all } \bar{v} \in H \subset V^{*}, \bar{u} \in V
$$

and we fix the Gelfand triple $V \subset H \subset V^{*}$.

\subsection{Monotone operators}

In this section, we introduce the matrix operator $A: W \rightarrow W \subset H \subset V^{*}$ defined by

$$
A(\bar{u}):=\left(\begin{array}{cc}
\operatorname{div} \mathbf{a}(\cdot, \nabla u) & 0 \\
-\tilde{u}|\tilde{u}|^{p-2} & -\mathbf{a}(\cdot, \nabla u) \cdot \nu
\end{array}\right) \quad \forall \bar{u} \in W .
$$

Given $\bar{u}:=(u, \tilde{u}), \bar{v}:=(v, \tilde{v}) \in W$ it holds

$$
\begin{aligned}
\left|V^{*}\langle A(\bar{u}), \bar{v}\rangle_{V}\right|= & \left|\langle A(\bar{u}), \bar{v}\rangle_{H}\right| \\
\leq & \left|\int_{\mathcal{O}} \operatorname{div} \mathbf{a}(x, \nabla u) v \mathrm{~d} x-\int_{\Gamma}(\mathbf{a}(\cdot, \nabla u) \cdot \nu) \tilde{v}(\xi) \mathrm{d} \xi\right| \\
& +\int_{\Gamma}|\tilde{u}(\xi)|^{p-1}|\tilde{v}(\xi)| \mathrm{d} \xi \\
= & \left|\int_{\mathcal{O}} \mathbf{a}(x, \nabla u) \cdot \nabla v \mathrm{~d} x\right|+\int_{\Gamma}|\tilde{u}(\xi)|^{p-1}|\tilde{v}(\xi)| \mathrm{d} \xi \\
\leq & \int_{\mathcal{O}}|\mathbf{a}(x, \nabla u(x))||\nabla v(x)| \mathrm{d} x+\int_{\Gamma}|\tilde{u}(\xi)|^{p-1}|\tilde{v}(\xi)| \mathrm{d} \xi .
\end{aligned}
$$


Applying the Leray-Lions conditions on a and Hölder's inequality we get

$$
\begin{gathered}
\int_{\mathcal{O}}|\mathbf{a}(x, \nabla u(x))||\nabla v(x)| \mathrm{d} x \leq \sigma\left(\|\rho\|_{L^{q}(\mathcal{O})}+\|\nabla u\|_{L^{p}(\mathcal{O})}^{p-1}\right)\|\nabla v\|_{L^{p}(\mathcal{O})} \\
\int_{\Gamma}|\tilde{u}(\xi)|^{p-1}|\tilde{v}(\xi)| \mathrm{d} \xi \leq\|\tilde{u}\|_{L^{p}(\Gamma)}^{p-1}\|\tilde{v}\|_{L^{p}(\Gamma) .}
\end{gathered}
$$

It follows that

$$
\left|V^{*}\langle A(\bar{u}), \bar{v}\rangle_{V}\right| \leq \sigma\left(\|\rho\|_{L^{q}(\mathcal{O})}+\|\bar{u}\|_{V}^{p-1}\right)\|\bar{v}\|_{V}
$$

and for all $\bar{u} \in W$

$$
\|A(\bar{u})\|_{V^{*}} \leq \sigma\left(\|\rho\|_{L^{q}(\mathcal{O})}+\|\bar{u}\|_{V}^{p-1}\right) .
$$

Therefore $A$ extends to a bounded nonlinear operator, again denoted by $A$, defined on all of $V$ and taking values in $V^{*}$ such that

$$
V^{*}\langle A(\bar{u}), \bar{v}\rangle_{V}=-\int_{\mathcal{O}} \mathbf{a}(x, \nabla u) \cdot \nabla v \mathrm{~d} x-\int_{\Gamma} \tilde{u}(\xi)|\tilde{u}(\xi)|^{p-2} \tilde{v}(\xi) \mathrm{d} \xi
$$

for all $\bar{u}, \bar{v} \in V$.

Theorem 4 The operator A satisfies the conditions of Hypothesis 1.

Proof. We divide the proof in four steps.

(H1) (Hemicontinuity): for all $\bar{u}, \bar{v}, \bar{x} \in V$ the map

$$
\mathbb{R} \ni \lambda \rightarrow V^{*}\langle A(\bar{u}+\lambda \bar{v}), \bar{x}\rangle_{V}
$$

is continuous.

For $\bar{u}, \bar{v}, \bar{w} \in V$, we have to show for $\lambda \in \mathbb{R},|\lambda| \leq 1, \lambda \rightarrow 0$ implies

$$
\begin{aligned}
&-\int_{\mathcal{O}}(\mathbf{a}(x, \nabla(u+\lambda v))-\mathbf{a}(x, \nabla u)) \cdot \nabla w \mathrm{~d} x \\
& \quad-\int_{\Gamma}\left[(\tilde{u}(\xi)+\lambda \tilde{v}(\xi))|\tilde{u}(\xi)+\lambda \tilde{v}(\xi)|^{p-2}-\tilde{u}(\xi)|\tilde{u}(\xi)|^{p-2}\right] \tilde{w}(\xi) \mathrm{d} \xi \longrightarrow 0 .
\end{aligned}
$$

The two integrands converge to zero as $\lambda \rightarrow 0$, $\mathrm{d} x$-a.e. ( $\mathrm{d} \xi$-a.e. respectively); in order to apply a dominated convergence theorem, we notice that, as $|\lambda| \leq 1$ and recalling assumption $(a 2)$, the integrands are dominated by

$$
\begin{aligned}
\mid(\mathbf{a}(x, \nabla(u+\lambda v)(x))- & \mathbf{a}(x, \nabla u(x))) \cdot \nabla w(x) \mid \\
\leq & 2 \sigma\left(|\rho(x)|+2^{p-1}\left(|\nabla u(x)|^{p-1}+|\nabla v(x)|^{p-1}\right)\right)|\nabla w(x)|
\end{aligned}
$$

and

$$
\begin{aligned}
|(\tilde{u}(\xi)+\lambda \tilde{v}(\xi))| \tilde{u}(\xi)+\left.\lambda \tilde{v}(\xi)\right|^{p-2}-\tilde{u}(\xi) \mid & \left.\tilde{u}(\xi)\right|^{p-2}|\tilde{w}(\xi)| \\
& \leq 2^{p-1}\left(|\tilde{u}(\xi)|^{p-1}+|\tilde{v}(\xi)|^{p-1}\right)|\tilde{w}(\xi)| ;
\end{aligned}
$$

note that these functions are $L^{1}(\mathcal{O})\left(L^{1}(\Gamma)\right.$ respectively) by Hölder's inequality. From Lebesgue's dominated convergence theorem, the claim follows. 
(H2) (Weak monotonicity): there exists $c \in \mathbb{R}$ such that for all $\bar{u}, \bar{v} \in V$ it holds

$$
V^{*}\langle A(\bar{u})-A(\bar{v}), \bar{u}-\bar{v}\rangle_{V} \leq c|\bar{u}-\bar{v}|_{H}^{2} .
$$

Let $\bar{u}, \bar{v} \in V$,

$$
\begin{aligned}
& V^{*}\langle A(\bar{u})-A(\bar{v}), \bar{u}-\bar{v}\rangle_{V}=-\int_{\mathcal{O}}(\mathbf{a}(x, \nabla u)-\mathbf{a}(x, \nabla v)) \cdot \nabla(u-v) \mathrm{d} x \\
&-\int_{\Gamma}\left(\tilde{u}(\xi)|\tilde{u}(\xi)|^{p-2}-\tilde{v}(\xi)|\tilde{v}(\xi)|^{p-2}\right)(\tilde{u}(\xi)-\tilde{v}(\xi)) \mathrm{d} \xi \leq 0
\end{aligned}
$$

since $\mathbf{a}$ is monotone (recall assumption (a3)) and the map $\mathbb{R} \ni t \rightarrow t|t|^{p-2}$ is increasing for all $p>2$.

(H3) (Coercivity): there exist $\left.c_{1} \in\right] 0, \infty\left[, c_{2} \in \mathbb{R}\right.$ and $p>2$ such that for all $\bar{v} \in V$ :

$$
V^{*}\langle A \bar{v}, \bar{v}\rangle_{V} \leq-c_{1}\|v\|_{V}^{p}+c_{2}|v|_{H}^{2} .
$$

By using condition (a1) for the Carathéodory function we have

$$
\begin{aligned}
V^{*}\langle A \bar{u}, \bar{u}\rangle_{V}=-\int_{\mathcal{O}} \mathbf{a}(x, \nabla u) \cdot \nabla u \mathrm{~d} x-\int_{\Gamma}|\tilde{u}(\xi)|^{p} \mathrm{~d} \xi & \\
& \leq-\delta \int_{\mathcal{O}}|\nabla u(x)|^{p} \mathrm{~d} x-\int_{\Gamma}|\tilde{u}(\xi)|^{p} \mathrm{~d} \xi .
\end{aligned}
$$

It follows from the equivalence of the norms that

$$
V^{*}\langle A \bar{u}, \bar{u}\rangle_{V} \leq-(1 \wedge \delta)\|u\|_{V}^{p}
$$

and coercivity holds with $\alpha=p$.

(H4) (Boundedness): there exists $c_{3} \in\left[0, \infty\left[\right.\right.$ and $c_{4} \in \mathbb{R}$ such that for all $\bar{v} \in V$ :

$$
\|A \bar{v}\|_{V^{*}} \leq c_{4}+c_{3}\|\bar{v}\|_{V}^{p-1} \text {. }
$$

It follows immediately from (3.4) for $\alpha=p, c_{3}=\sigma$ and $c_{4}=\sigma\|\rho\|_{L^{q}(\mathcal{O})}$.

\subsection{Stochastic forcing}

Let $(\Omega, \mathcal{F}, \mathbb{P})$ be a probability space with a filtration $\left(\mathcal{F}_{t}\right)$. On the Hilbert space $H$, we define a standard cylindrical Wiener process $W(t)=(w(t), \tilde{w}(t))$, i.e., formally we have

$$
W(t)=\sum_{i=1}^{2} \sum_{k=1}^{\infty} \beta_{k}^{i}(t) g_{k}^{i}
$$

where $\left\{g_{k}^{1}\right\}$ and $\left\{g_{k}^{2}\right\}$ are orthonormal basis in $L^{2}(\mathcal{O})$ and $L^{2}(\Gamma)$ respectively, $\left\{\beta_{k}^{i}\right\}$ is a (double indices) sequence of independent real valued standard Brownian motions on $(\Omega, \mathcal{F}, \mathbb{P})$ adapted to $\left(\mathcal{F}_{t}\right)$. 
Notice that $W(t)$ is not a stochastic process with values in $H$, since the series diverges almost surely. However, we further introduce $B \in L_{2}(H)$ as the matrixoperator

$$
B:=\left(\begin{array}{cc}
b & 0 \\
0 & \tilde{b}
\end{array}\right)
$$

Then $B W(t)$ is a Gaussian process with values in $H$, with covariance operator $B B^{\star}$ in $H$. For more details, we refer for instance to $[6, \S 2.3 .2]$.

\subsection{Existence and uniqueness of the solution}

With the aid of the operators $A$ and $B$ introduced in (3.5) and (3.6) we rewrite system (3.1) as a nonlinear SDE of the form

$$
\left\{\begin{aligned}
\mathrm{d} \bar{u}(t) & =A(\bar{u}(t)) \mathrm{d} t+B \mathrm{~d} W(t) \\
\bar{u}(0) & =\bar{u}_{0} .
\end{aligned}\right.
$$

We shall use the general theory recalled in Section 2 in order to prove the existence of a solution of (3.7).

Theorem 5 Let $\bar{u}_{0} \in H, A$ be given by (3.5) with the Carathéodory function a satisfying the Leray-Lions conditions (a1)-(a3), $B \in L_{2}(H)$ be given by (3.6) and $T \in[0, \infty)$ be fixed. Then there exists a unique variational solution $\{X(t), t \in$ $[0, T]\}$ of (3.7) and it holds, further, that

$$
\mathbb{E} \sup _{t \in[0, T]}|\bar{u}(t)|_{H}^{2}<\infty .
$$

Moreover, the solution is a Markov process.

The proof follows from Theorem 3 , since by Theorem 4 the operator $A$ satisfies (H1)-(H4) and we assume $B \in L_{2}(H)$.

\section{Invariant measures for the stochastic equation}

We consider the abstract equation (3.7) under the assumptions introduced in previous section. We denote by $\bar{u}\left(t ; \bar{u}_{0}\right)$ the solution at time $t$ with initial condition $\bar{u}_{0}$ at time $t=0$. Let $P_{t}$ be the transition semigroup defined by

$$
P_{t} \varphi\left(\bar{u}_{0}\right)=\mathbb{E}\left[\varphi\left(\bar{u}\left(t ; \bar{u}_{0}\right)\right)\right]
$$

for $\varphi \in C_{b}(H)$ the space of bounded and continuous functions on $H$. We show by means of the Krylov-Bogoliubov's theorem (see e.g. [5, Theorem 1.11] for details) that $P_{t}$ admits an invariant measure $\mu$, i.e. a Borel probability measure on $H$ such that

$$
\int_{H} P_{t} \varphi(u) \mu(\mathrm{d} u)=\int_{H} \varphi(u) \mu(\mathrm{d} u) \quad \forall \varphi \in C_{b}(H), t>0 .
$$

By the Lebesgue's dominated convergence theorem this property can be extended to the space of $\mu$-integrable functions. 
Remark 2 Note that the solution to (3.7) generates a Markovian semigroup $P_{t}$ on $B_{b}(H)$ by the usual prescription (4.1). The continuity of the solution map ensures that $P_{t}$ is Feller, i.e. $P_{t} \varphi \in C_{b}(H)$ for any $\varphi \in C_{b}(H)$, for all $t \geq 0$, see e.g. [5, §1.2.5].

Theorem 6 There exists an invariant measure $\mu$ for the transition semigroup $P_{t}$ associated with (3.7). Moreover, $\mu$ is concentrated on $V$, i.e. $\mu(V)=1$.

Proof. We apply Itô formula (compare [17, Theorem 4.2.5] and [12, Theorem I.3.1]) to (3.7) to get

$$
\mathbb{E}\left|\bar{u}\left(t ; \bar{u}_{0}\right)\right|_{H}^{2}=\left|\bar{u}_{0}\right|_{H}^{2}+2 \mathbb{E} \int_{0}^{t} V^{*}\left\langle A\left(\bar{u}\left(s ; \bar{u}_{0}\right)\right), \bar{u}\left(s ; \bar{u}_{0}\right)\right\rangle_{V} \mathrm{~d} s+t \operatorname{Tr}\left(B B^{*}\right)
$$

which, taking into account the coercivity condition $(H 3)$, becomes

$$
\begin{aligned}
\mathbb{E}\left|\bar{u}\left(t ; \bar{u}_{0}\right)\right|_{H}^{2} \leq\left|\bar{u}_{0}\right|_{H}^{2}-2 c_{1} \mathbb{E} \int_{0}^{t} \| & \bar{u}\left(s ; \bar{u}_{0}\right) \|_{V}^{p} \mathrm{~d} s \\
& +2 c_{2} \mathbb{E} \int_{0}^{t}\left|\bar{u}\left(s ; \bar{u}_{0}\right)\right|_{H}^{2} \mathrm{~d} s+t \operatorname{Tr}\left(B B^{*}\right) .
\end{aligned}
$$

Notice that by the continuous inclusion $V \hookrightarrow H$ there exists a constant $k$ such that $|\bar{v}|_{H} \leq k\|\bar{v}\|_{V}$ for all $\bar{v} \in V$, therefore

$$
\begin{aligned}
\mathbb{E}\left|\bar{u}\left(t ; \bar{u}_{0}\right)\right|_{H}^{2} \leq\left|\bar{u}_{0}\right|_{H}^{2}-\frac{2 c_{1}}{k^{p}} \mathbb{E} \int_{0}^{t}\left|\bar{u}\left(s ; \bar{u}_{0}\right)\right|_{H}^{p} \mathrm{~d} s & \\
& +2 c_{2} \mathbb{E} \int_{0}^{t}\left|\bar{u}\left(s ; \bar{u}_{0}\right)\right|_{H}^{2} \mathrm{~d} s+t \operatorname{Tr}\left(B B^{*}\right) .
\end{aligned}
$$

In the same way we obtain also the differential inequality

$$
\frac{\mathrm{d}}{\mathrm{d} t} \mathbb{E}\left|\bar{u}\left(t ; \bar{u}_{0}\right)\right|_{H}^{2} \leq-\frac{2 c_{1}}{k^{p}} \mathbb{E}\left|\bar{u}\left(t ; \bar{u}_{0}\right)\right|_{H}^{p}+2 c_{2} \mathbb{E}\left|\bar{u}\left(t ; \bar{u}_{0}\right)\right|_{H}^{2}+\operatorname{Tr}\left(B B^{*}\right) .
$$

Since $p>2$ by assumption, it follows that $|\varepsilon x|^{2} \leq|\varepsilon x|^{p}+1$ for any $\varepsilon>0$ and then $-|x|^{p} \leq-\varepsilon^{2-p}|x|^{2}+\varepsilon^{-p}$. This implies that

$$
\begin{aligned}
\frac{\mathrm{d}}{\mathrm{d} t} \mathbb{E}\left|\bar{u}\left(t ; \bar{u}_{0}\right)\right|_{H}^{2} & \leq-\frac{2 c_{1}}{\varepsilon^{p-2} k^{p}} \mathbb{E}\left|\bar{u}\left(t ; \bar{u}_{0}\right)\right|_{H}^{2}+\frac{2 c_{1}}{\varepsilon^{p} k^{p}}+2 c_{2} \mathbb{E}\left|\bar{u}\left(t ; \bar{u}_{0}\right)\right|_{H}^{2}+\operatorname{Tr}\left(B B^{*}\right) \\
& =-\left(\frac{2 c_{1}}{\varepsilon^{p-2} k^{p}}-2 c_{2}\right) \mathbb{E}\left|\bar{u}\left(t ; \bar{u}_{0}\right)\right|_{H}^{2}+\frac{2 c_{1}}{\varepsilon^{p} k^{p}}+\operatorname{Tr}\left(B B^{*}\right) .
\end{aligned}
$$

Choosing a suitable $\varepsilon>0$ such that $\gamma:=\frac{2 c_{1}}{\varepsilon^{p-2} k^{p}}-2 c_{2}>0$ we can apply Gronwall's lemma and conclude that there exists a constant $C$ independent of $t$ such that

$$
\mathbb{E}\left|\bar{u}\left(t ; \bar{u}_{0}\right)\right|_{H}^{2} \leq e^{-\gamma t}\left|\bar{u}_{0}\right|_{H}^{2}+C \quad \text { for all } t \geq 0 .
$$

Now by (4.2) and (4.3) it follows that

$$
\mathbb{E} \int_{0}^{t}\left\|\bar{u}\left(s ; \bar{u}_{0}\right)\right\|_{V}^{p} \mathrm{~d} s \leq \frac{1}{2 c_{1}}\left[\left(1+\frac{2 c_{2}}{\gamma}\right)\left|\bar{u}_{0}\right|_{H}^{2}+t\left(2 c_{2} C+\operatorname{Tr}\left(B B^{*}\right)\right)\right] .
$$


To apply the Krylov-Bogoliubov's theorem we have to verify that for some $\bar{u}_{0} \in H$ the set of probability measures $\left(\mu_{T}\right)_{T>0}$ is tight, where

$$
\mu_{T}(E):=\frac{1}{T} \int_{0}^{T} \pi_{t}\left(\bar{u}_{0}, E\right) \mathrm{d} t \quad \text { for all } E \in \mathcal{B}(H) \text { and } T>0,
$$

and $\pi_{t}\left(\bar{u}_{0}, \cdot\right)$ is the law of the random variable $\bar{u}\left(t ; \bar{u}_{0}\right)$. We prove tightness for $\left(\mu_{T}\right)_{T>0}$ with $\bar{u}_{0}=0$ by means of the Prokhorov's theorem (see $[6, \S 2.1]$ ).

Let us consider the closed balls

$$
B_{0, n}:=\left\{\bar{v} \in H:\|\bar{v}\|_{V} \leq n\right\}
$$

for $n \in \mathbb{N}$, which are bounded in $V$. Thanks to the compact imbedding $V \hookrightarrow H$ it results that $B_{0, n}$ is a compact subset of $H$, then we have to show that

$$
\lim _{n \rightarrow+\infty} \mu_{T}\left(B_{0, n}^{c}\right)=0 \quad \text { uniformly in } T>0 .
$$

By Markov's inequality we get

$$
\begin{aligned}
\mu_{T}\left(B_{0, n}^{c}\right) & =\frac{1}{T} \int_{0}^{T} \pi\left(t, B_{0, n}^{c}\right) \mathrm{d} t \\
& =\frac{1}{T} \int_{0}^{T} \mathbb{P}\left(\left\|\bar{u}\left(t ; \bar{u}_{0}\right)\right\|_{V} \geq n\right) \mathrm{d} t \leq \frac{1}{T} \int_{0}^{T} \frac{\mathbb{E}\left\|\bar{u}\left(t ; \bar{u}_{0}\right)\right\|_{V}^{p}}{n^{p}} \mathrm{~d} t .
\end{aligned}
$$

By (4.4) it follows that for $n \rightarrow+\infty$

$$
\frac{1}{T} \int_{0}^{T} \pi\left(t, B_{0, n}^{c}\right) \mathrm{d} t \leq \frac{1}{2 c_{1} T n^{p}}\left[\left(1+\frac{2 c_{2}}{\gamma}\right)\left|\bar{u}_{0}\right|_{H}^{2}+T\left(2 c_{2} C+\operatorname{Tr}\left(B B^{*}\right)\right)\right] \rightarrow 0
$$

uniformly in $T>0$.

Therefore, by the Krylov-Bogoliubov's theorem there exists a subsequence $\left(T_{k}\right)_{k \in \mathbb{N}}$ such that $\mu_{T_{k}} \rightarrow \mu$ weakly, where $\mu$ is an invariant measure for $P_{t}$.

Next, we show that $\mu$ verifies $\mu(V)=1$. Let us define the function $\Theta(x):=$ $\sup _{i \in \mathbb{N}}\left|\left\langle\ell_{i}, x\right\rangle\right|$, where $\left\{\ell_{i}\right\}_{i \in \mathbb{N}}$ is a countable dense subset of $B_{1}^{V^{*}} \cap H$ in the topology of $H$, and $B_{1}^{V^{*}}$ is the closed unit ball in $V^{*}$. It follows, taking into account [17, Ex. 4.2.3], that $\Theta: H \rightarrow[0, \infty]$ is a lower semicontinuous function such that

$$
\Theta(x)= \begin{cases}\|x\|_{V}, & x \in V, \\ +\infty, & x \in H \backslash V .\end{cases}
$$

Recalling that (4.4) implies

$$
\int_{H}\|x\|_{V}^{p} \mu_{T_{k}}(\mathrm{~d} x)=\frac{1}{T_{k}} \int_{0}^{T_{k}} \mathbb{E}\|\bar{u}(s ; 0)\|_{V}^{p} \mathrm{~d} s \leq \frac{1}{2 c_{1}}\left[2 c_{2} C+\operatorname{Tr}\left(B B^{*}\right)\right]<\infty,
$$


uniformly with respect to $T_{k}$, it holds that

$$
\begin{aligned}
\int_{H} \Theta(x)^{p} \mu(\mathrm{d} x) & =\lim _{N \rightarrow \infty} \lim _{M \rightarrow \infty} \int_{H}\left(\sup _{i \leq N}\left|\left\langle\ell_{i}, x\right\rangle\right|^{p} \wedge M\right) \mu(\mathrm{d} x) \\
& =\sup _{N, M \in \mathbb{N}} \lim _{k \rightarrow \infty} \int_{H}\left(\sup _{i \leq N}\left|\left\langle\ell_{i}, x\right\rangle\right|^{p} \wedge M\right) \mu_{T_{k}}(\mathrm{~d} x) \\
& \leq \liminf _{k \rightarrow \infty} \sup _{N, M \in \mathbb{N}} \int_{H}\left(\sup _{i \leq N}\left|\left\langle\ell_{i}, x\right\rangle\right|^{p} \wedge M\right) \mu_{T_{k}}(\mathrm{~d} x) \\
& =\liminf _{k \rightarrow \infty} \int_{H}\|x\|_{V}^{p} \mu_{T_{k}}(\mathrm{~d} x)<\infty .
\end{aligned}
$$

Hence $\Theta<\infty \mu$-a.e., thus also $\mu(V)=1$ since $\{x \in H: \Theta(x)<\infty\}=V$.

We have just proved that there exists an invariant measure $\mu$ for $P_{t}$, obtained thanks to Krylov-Bogoliubov's theorem, which is concentrated on $V$; for this, we have made use of the fact that it is constructed as weak limit of a particular sequence of measures. The next theorem asserts that any invariant measure for $P_{t}$ is concentrated on $V$.

Theorem 7 Let $\mu$ be an invariant measure for $P_{t}$. Then $\mu$ satisfies the estimate

$$
\int_{H}\left(|x|_{H}^{2}+\|x\|_{V}^{p}\right) \mu(\mathrm{d} x)<\infty .
$$

Proof. Let $\mu$ be an invariant measure for $P_{t}$. As a first step, we show that $\mu$ has finite second moment. Let $\varphi(x)=|x|_{H}^{2}$; we consider the bounded smooth approximation of $|\cdot|_{H}^{2}$ defined by:

$$
\varphi_{\delta}(x)=\frac{|x|_{H}^{2}}{1+\delta|x|_{H}^{2}}, \quad \delta>0 .
$$

It is $\varphi_{\delta}=g_{\delta} \circ \varphi$, where the function $g_{\delta}(y)=\frac{y}{1+\delta y}$ is such that $g_{\delta} \in C_{b}^{1}\left(\mathbb{R}^{+}, \mathbb{R}\right)$. It holds $\left|\operatorname{Tr}\left(B D^{2} \varphi_{\delta}(x) B^{*}\right)\right|_{L(H)} \leq C$ and $\varphi_{\delta}(x) \rightarrow \varphi(x)=|x|_{H}^{2}$ when $\delta \rightarrow 0$. We apply Itô's formula to $\varphi_{\delta}$ and, by using the coercivity condition $(H 3)$ for $A$, it follows

$$
\begin{aligned}
\mathbb{E}\left(\frac{|\bar{u}(t, x)|_{H}^{2}}{1+\delta|\bar{u}(t, x)|_{H}^{2}}\right)+ & 2 c_{1} \mathbb{E}\left(\int_{0}^{t} \frac{\|\bar{u}(s, x)\|_{V}^{p}}{\left(1+\delta|\bar{u}(s, x)|_{H}^{2}\right)^{2}} \mathrm{~d} s\right) \\
& \leq \frac{|x|_{H}^{2}}{1+\delta|x|_{H}^{2}}+2 c_{2} \mathbb{E}\left(\int_{0}^{t} \frac{|\bar{u}(s, x)|_{H}^{2}}{\left(1+\delta|\bar{u}(s, x)|_{H}^{2}\right)^{2}} \mathrm{~d} s\right)+t C .
\end{aligned}
$$

We apply once more the inequalities $|x|^{2} \leq \varepsilon^{p-2}|x|^{p}+\varepsilon^{-2}$ and $|x|_{H} \leq k\|x\|_{V}$ : then we have

$$
\frac{\varepsilon^{2-p}}{k^{2}}|x|_{H}^{2} \leq\|x\|_{V}^{p}+\varepsilon^{-p}
$$


hence

$$
\begin{gathered}
\mathbb{E}\left(\frac{|\bar{u}(t, x)|_{H}^{2}}{1+\delta|\bar{u}(t, x)|_{H}^{2}}\right)+2 c_{1} \frac{\varepsilon^{2-p}}{k^{2}} \mathbb{E}\left(\int_{0}^{t} \frac{|\bar{u}(s, x)|_{H}^{2}}{\left(1+\delta|\bar{u}(s, x)|_{H}^{2}\right)^{2}} \mathrm{~d} s\right) \\
\leq \frac{|x|_{H}^{2}}{1+\delta|x|_{H}^{2}}+2 c_{2} \mathbb{E}\left(\int_{0}^{t} \frac{|\bar{u}(s, x)|_{H}^{2}}{\left(1+\delta|\bar{u}(s, x)|_{H}^{2}\right)^{2}} \mathrm{~d} s\right)+t\left(C+2 c_{1} \varepsilon^{-p}\right) .
\end{gathered}
$$

At this point, we can integrate with respect to $\mu$ on $H$ since all functions are integrable with respect to $\mu$; using the definition of invariant measure, we obtain, by choosing $t=1$

$$
\begin{aligned}
& 2 c_{1} \frac{\varepsilon^{2-p}}{k^{2}} \int_{H} \frac{|x|_{H}^{2}}{\left(1+\delta|x|_{H}^{2}\right)^{2}} \mu(\mathrm{d} x) \\
& \leq 2 c_{2} \int_{H} \frac{|x|_{H}^{2}}{\left(1+\delta|x|_{H}^{2}\right)^{2}} \mu(\mathrm{d} x)+\left(C+2 c_{1} \varepsilon^{-p}\right) .
\end{aligned}
$$

hence

$$
2\left(c_{1} \frac{\varepsilon^{2-p}}{k^{2}}-c_{2}\right) \int_{H} \frac{|x|_{H}^{2}}{\left(1+\delta|x|_{H}^{2}\right)^{2}} \mu(\mathrm{d} x) \leq\left(C+2 c_{1} \varepsilon^{-p}\right)
$$

and we can choose $\varepsilon$ large enough and independent of $\delta$ such that

$$
\int_{H} \frac{|x|_{H}^{2}}{\left(1+\delta|x|_{H}^{2}\right)^{2}} \mu(\mathrm{d} x)<C
$$

uniformly on $\delta$ for some constant $C>0$. Letting $\delta \rightarrow 0$, by the Monotone Convergence Theorem we get the first part of (4.5)

$$
\int_{H}|x|_{H}^{2} \mu(\mathrm{d} x)<\infty
$$

Now we consider the mapping $\psi: x \in H \mapsto\|x\|_{V}^{p}$ and we prove that $\psi \in$ $L^{1}(H ; \mu)$.

Let us define $\psi_{N}(x)=\|x\|_{V}^{p} \wedge N$ for $N \in \mathbb{N}$; notice that $\Psi_{N} \in B_{b}(H)$; then, by the very definition of invariant measure, Tonelli's theorem and (4.4) it holds

$$
\begin{aligned}
\sup _{N \in \mathbb{N}} \int_{H} \psi_{N}(x), \mu(\mathrm{d} x) & =\sup _{N \in \mathbb{N}} \int_{0}^{1} \int_{H} P_{s} \psi_{N}(x) \mathrm{d} s \mu(\mathrm{d} x) \\
& =\sup _{N \in \mathbb{N}} \int_{H} \int_{0}^{1} \mathbb{E}\left[\|\bar{u}(s ; 0, x)\|_{V}^{p} \wedge N\right] \mathrm{d} s \mu(\mathrm{d} x) \\
& \leq \int_{H} \int_{0}^{1} \mathbb{E}\|\bar{u}(s ; 0, x)\|_{V}^{p} \mathrm{~d} s \mu(\mathrm{d} x) \\
& \leq C \int_{H}\left(|x|_{H}^{2}+1\right) \mu(\mathrm{d} x)
\end{aligned}
$$


uniformly on $N$ for some constant $C>0$. Taking into account (4.6) we get

$$
\sup _{N \in \mathbb{N}} \int_{H} \psi_{N}(x) \mu(\mathrm{d} x) \leq C
$$

and again by means of the Monotone Convergence Theorem

$$
\int_{H}\|x\|_{V}^{p} \mu(\mathrm{d} x)<\infty
$$

Corollary 8 There exists an ergodic invariant measure for the semigroup $P_{t}$.

Proof. This result is based on the well known Krein-Milman theorem (see e.g. [2, Theorem 7.68]) about the characterization of convex compact sets in locally convex Hausdorff spaces as closed convex hull of its extreme points. In particular (see e.g. [2, Theorem 19.25]) it results that for an arbitrary Markov transition semigroup $P_{t}$, the ergodic measures are precisely the extreme points of the (possibly empty) convex set of its invariant measures.

Now let us denote by $\mathcal{P}(H)$ the space of probability measures on $H$ and by $\Lambda \subset \mathcal{P}(H)$ the convex set of all invariant measures for the Markov semigroup $P_{t}$. We have just proved that $\Lambda$ is nonempty, then we have to show its closure is compact or equivalently $\Lambda$ is tight. In such a way the convex hull of extreme points of $\Lambda$ is not empty as much as the set of ergodic measures. By previous theorem we conclude that there exists a constant $K$ independent of $\mu \in \Lambda$ such that

$$
\int_{H}\|x\|_{V}^{p} \mu(\mathrm{d} x)<K
$$

By Markov's inequality

$$
\sup _{\mu \in \Lambda} \mu\left(B_{0, n}^{c}\right)=\sup _{\mu \in \Lambda} \mu\left\{x \in H \mid\|x\|_{V}>n\right\} \leq \sup _{\mu \in \Lambda} \frac{1}{n^{p}} \int_{H}\|x\|_{V}^{p} \mu(\mathrm{d} x) \leq \sup _{\mu \in \Lambda} \frac{K}{n^{p}} \rightarrow 0
$$

as $n \rightarrow \infty$. We have thus proved that $\Lambda$ is tight and has extreme points which are ergodic invariant measures for $P_{t}$.

In order to prove uniqueness of an invariant measure $\mu$ we need an extra superlinearity assumption on the operator $A$ (see e.g. [3, Hypothesis 1.2]).

Theorem 9 Assume, instead of (H2), that there exist constants $c>0$ and $\varepsilon>0$ such that

$$
V^{*}\langle A(u)-A(v), u-v\rangle_{V} \leq c|u-v|_{H}^{2+\varepsilon} \quad \forall u, v \in V .
$$

Then the transition semigroup $P_{t}$ associated with (2.1) has a unique strongly mixing invariant measure $\mu$ such that

$$
\left|P_{t} \varphi(x)-\int_{H} \varphi(y) \mu(\mathrm{d} y)\right| \leq c\|D \varphi\|_{\infty}, \quad t \geq 0,
$$

for all $\varphi \in C_{b}^{1}(H)$. 
Remark 3 Note that in our case assumption (4.7) reduces to

$$
(\mathbf{a}(x, \xi)-\mathbf{a}(x, \eta)) \cdot(\xi-\eta) \geq c|\xi-\eta|^{2+\varepsilon} \quad \text { for a.e. } x \in \mathcal{O} \text { and for all } \xi \neq \eta \in \mathbb{R}^{n} .
$$

This condition is fulfilled, for instance, in the special case where $\mathbf{a}(x, \xi)=|\xi|^{p-2} \xi$ for $p>2$ which means that the internal dynamic is governed by a p-Laplacian.

Proof. We give a sketch of the proof, compare also [3] for details.

Let $x, y \in H$, by Itô's formula for the square of the norm, (4.7) and Jensen's inequality we obtain

$$
\frac{\mathrm{d}}{\mathrm{d} t} \mathbb{E}|u(t ; x)-u(t ; y)|_{H}^{2} \leq-c\left(\mathbb{E}|u(s ; x)-u(s ; y)|_{H}^{2}\right)^{1+\varepsilon / 2}
$$

which implies $\mathbb{E}|u(t ; x)-u(t ; y)|_{H}^{2} \rightarrow 0$ as $t \rightarrow \infty$. Now let $\mu$ be an invariant measure for $P_{t}$, then

$$
\left|P_{t} \varphi(x)-\int_{H} \varphi(y) \mu(\mathrm{d} y)\right| \leq\|D \varphi\|_{\infty} \int_{H} \mathbb{E}|u(t ; x)-u(t ; y)|_{H}^{2} \mu(\mathrm{d} y) \quad \forall \varphi \in C_{b}^{1}(H) .
$$

Since $C_{b}^{1}(H) \subset L^{2}(H, \mu)$ densely, it holds

$$
\lim _{t \rightarrow \infty} P_{t} \varphi(x)=\int_{H} \varphi(y) \mu(\mathrm{d} y), \quad \forall \varphi \in L^{2}(H, \mu)
$$

i.e. $\mu$ is ergodic and strongly mixing.

\section{References}

[1] R. A. Adams and J. J. F. Fournier. Sobolev spaces, volume 140 of Pure and Applied Mathematics (Amsterdam). Elsevier/Academic Press, Amsterdam, second edition, 2003.

[2] C. D. Aliprantis and K. C. Border. Infinite dimensional analysis. Springer, Berlin, third edition, 2006. A hitchhiker's guide.

[3] V. Barbu and G. Da Prato. Ergodicity for nonlinear stochastic equations in variational formulation. Appl. Math. Optim., 53(2):121-139, 2006.

[4] I. Chueshov and B. Schmalfuss. Parabolic stochastic partial differential equations with dynamical boundary conditions. Differential Integral Equations, 17(7-8):751-780, 2004.

[5] G. Da Prato. Kolmogorov equations for stochastic PDEs. Birkhäuser Verlag, Basel, 2004.

[6] Giuseppe Da Prato and Jerzy Zabczyk. Stochastic equations in infinite dimensions, volume 44 of Encyclopedia of Mathematics and its Applications. Cambridge University Press, Cambridge, 1992.

[7] Françoise Demengel and Gilbert Demengel. Espaces fonctionnels. Savoirs Actuels (Les Ulis). [Current Scholarship (Les Ulis)]. EDP Sciences, Les Ulis, 2007. Utilisation dans la résolution des équations aux dérivées partielles. [Application to the solution of partial differential equations].

[8] J. I. Díaz and L. Tello. A nonlinear parabolic problem on a Riemannian manifold without boundary arising in climatology. Collect. Math., 50(1):19-51, 1999. 
[9] J. Duan and D. Yang. An Impact of Stochastic Dynamic Boundary Conditions on the Evolution of the Cahn-Hilliard System. Stochastic Analysis and Applications, 25:613-639, 2007.

[10] Jinqiao Duan, Hongjung Gao, and Björn Schmalfuß. Stochastic dynamics of a coupled atmosphere-ocean model. Stoch. Dyn., 2(3):357-380, 2002. Special issue on stochastic climate models.

[11] E. Gagliardo. Caratterizzazioni delle tracce sulla frontiera relative ad alcune classi di funzioni in $n$ variabili. Rend. Sem. Mat. Univ. Padova, 27:284-305, 1957.

[12] N. V. Krylov and B. L. Rozovskiǔ. Stochastic evolution equations. In Current problems in mathematics, Vol. 14 (Russian), pages 71-147, 256. Akad. Nauk SSSR, Vsesoyuz. Inst. Nauchn. i Tekhn. Informatsii, Moscow, 1979.

[13] J. Leray and J.-L. Lions. Quelques résultats de Višik sur les problèmes elliptiques non linéaires par les méthodes de Minty-Browder. Bull. Soc. Math. France, 93:97-107, 1965.

[14] J.-L. Lions. Quelques méthodes de résolution des problèmes aux limites non linéaires. Dunod, 1969.

[15] C. Marinelli and G. Ziglio. Ergodicity for nonlinear stochastic evolution equations with multiplicative Poisson noise. Dynamics of Partial Differential Equations, $7(1): 1-24,2010$.

[16] P. Müller. Stochastic forcing of oceanic motions. In Stochastic models in geosystems (Minneapolis, MN, 1994), volume 85 of IMA Vol. Math. Appl., pages 219-237. Springer, New York, 1997.

[17] C. Prévôt and M. Röckner. A concise course on stochastic partial differential equations, volume 1905 of Lecture Notes in Mathematics. Springer, Berlin, 2007.

[18] R. E. Showalter. Monotone operators in Banach space and nonlinear partial differential equations, volume 49. American Mathematical Society, Providence, RI, 1997.

[19] N. Su. Multidimensional degenerate diffusion problem with evolutionary boundary condition: existence, uniqueness, and approximation. In Flow in porous media (Oberwolfach, 1992), volume 114 of Internat. Ser. Numer. Math., pages 165-178. Birkhäuser, Basel, 1993.

[20] I. I. Vrabie. $C_{0}$-semigroups and applications. North-Holland, Amsterdam, 2003. 